\title{
Bringing models to life
}

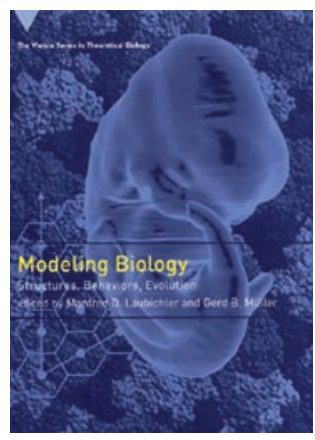

\section{Modeling Biology}

Edited by Manfred D. Laublicher and Gerd B. Müller

MIT Press, $2007 \bullet \$ 50.00 / £ 32.95$

\section{Pierre-François Lenne}

Scientific models are generally defined as frameworks that capture basic mechanistic connections between key parameters and predict behaviour quantitatively. Building models to explain and ultimately understand living systems in their complexity is one of the major goals of contemporary biology. More than simple animations, imitations or mathematical formalizations, models are unique tools that may allow the development of intelligible theories about biological phenomena. In this context, Modeling Biology offers a vast and in depth exploration of current models and modelling practices in biology. The book gathers the contributions of experts from different fields in biology, such as morphology, development, behaviour and evolution, as well as, and perhaps more surprisingly, from the philosophy and history of sciences. Having grown out of a theory-oriented workshop and enriched by additional perspectives, the book reinforces the need to bring together biologists, mathematicians, physicists and philosophers.

This patchwork of contributions exemplifies the idea that biological sciences use a variety of models spanning different scales and levels of complexity. In this respect, the editors of the book, Manfred D. Laublicher and Gerd B. Müller, are successful in presenting an eclectic range of models from molecular structures to whole organisms, which, despite their differences, share common multi-scale approaches combining material and conceptual models.

The book begins with a discussion of conceptual issues, asking what are models, what do they contain and what strategies can be used to model relations and interactions between parts of biological systems. By addressing these questions, the authors provide a short but essential philosophical and historical perspective on models and on the nature and function of explanation in biology. These concerns are discussed to differing degrees in the introduction and discussion of most of the following chapters.

Pierre-François Lenne is at the Fresnel Institute, CNRS and Aix-Marseille University, Domaine Universitaire de Saint Jérôme, 13397, Marseille, France.e-mail: lenne@fresnel.fr
The next part focuses on biological forms and includes models of molecular, cellular and organismal morphology. In this field, the analysis of geometric constraints and physical laws are used to reveal the organizational principles of forms found in nature. The book then explores the realm of developmental biology, where models fulfil representational, analytical and heuristic roles. In contrast to models on morphology, which are mostly static, models in developmental biology integrate dynamic aspects of the phenomena under study at different levels. The four chapters devoted to development underscore the links between gene regulation, cell-cell and cell-environment interactions. They explain how these interactions can produce complex phenomena such as neural growth, collective movements of cells, or organ asymmetries. The study of physical properties and behaviour of cells during development provide not only theoretical models, to be tested with careful experimentation, but also predictions of new phenomena, which can escape the scrutiny of experimental biologists.

In the section on behaviour, the reader is introduced to a new modelling dimension, where robot-based models, for which underlying principles are known, offer new ways to study the emergence of behaviours. The last section of the book is an insightful presentation of evolution. In this field, models have always been important because history does not lend itself to experimental tests. Specific modelling and test strategies have thus been developed. The first two chapters in this section focus on models of plant evolution and behaviour of social insects to illustrate the concepts of optimization and emergence. The last chapter explores the role of modelling in the new interdisciplinary field of evolutionary developmental biology (evo-devo), which aims to integrate development with evolution and, increasingly, with ecology. The authors argue that in the future, explaining patterns of phenotypic evolution should rely on a predictive and experimentally testable framework incorporating current material and conceptual models.

This book is a valuable resource for students in biology, physics, mathematics and philosophy, interested in learning about what modelling can bring to the life sciences and how models are elaborated. Most chapters are well explained and documented with the necessary, but never tedious, mathematical formalism. Because of the variety of models presented, some chapters should also provide a good read for experts. Finally, by elaborating on the success of models in providing predictions and explaining observations, the authors aim to dispel scepticism about models and encourage experimental biologists to use them. One can hope that readers will take time to consider their choice of a particular experimental model, the conceptual tracks they are following, and perhaps ponder on what their data could gain from a modelling perspective. 